tions, The Rice Institute Pamphlet, vol. 29, 1942.

3. R. Paley and N. Wiener, Fourier transforms in the complex domain, New York, 1934.

4. L. Schwartz, Etudes des sommes d'exponentielles reelles, Paris, 1943.

5. E. Titchmarsh, The theory of functions, 2d ed., Oxford, 1939.

HARVARD UNIVERSITY

\title{
ON THE ABSOLUTE CONVERGENCE OF TRIGONOMETRICAL SERIES
}

TAMOTSU TSUCHIKURA AND SHIGEKI YANO

1. Theorem 1. Suppose that a trigonometrical series

$$
\sum \rho_{n} \cos \left(n x-\alpha_{n}\right) \quad\left(\rho_{n} \geqq 0, n=1,2, \cdots\right)
$$

and its conjugate series

$$
\sum \rho_{n} \sin \left(n x-\alpha_{n}\right)
$$

are convergent absolutely at $x=x_{0}$ and $x=x_{1}$, respectively. If

$$
x_{1}-x_{0}=p \pi / q \quad(p / q \text { irreducible })
$$

where $p$ is an integer positive, negative, or zero, and $q$ is an odd integer, then

$$
\sum \rho_{n}<\infty \text {. }
$$

We shall see in Theorem 2 that the above theorem is no longer true if $p / q$ is replaced by $p^{\prime} / q^{\prime}$ with an even $q^{\prime}$ and $p^{\prime} \neq 0$, or by an irrational number.

Proof of Theorem 1. If we put $x_{1}-x_{0}=p \pi / q \equiv h$, then by Fatou's theorem $^{1}$

$$
\sum \rho_{n}\left|\cos \left(n\left(x_{1}-s h\right)-\alpha_{n}\right)\right|<\infty
$$

for every odd $s$. Hence from the identity

$$
\begin{aligned}
\sin \left(n x_{1}-\alpha_{n}\right)= & \cos n s h \sin \left(n\left(x_{1}-s h\right)-\alpha_{n}\right) \\
& +\sin n s h \cos \left(n\left(x_{1}-s h\right)-\alpha_{n}\right)
\end{aligned}
$$

we deduce immediately that

$$
\left.\sum \rho_{n} \mid \cos n s h \sin \left(n\left(x_{1}-s h\right)\right)-\alpha_{n}\right) \mid<\infty .
$$

Received by the editors October 18, 1948 and, in revised form, May 14, 1949.

1 See, for example, A. Zygmund, Trigonometrical series, p. 134. 
On the other hand from (1) we have

$$
\sum \rho_{n}\left|\cos n s h \cos \left(n\left(x_{1}-s h\right)-\alpha_{n}\right)\right|<\infty,
$$

hence from (2) and (3)

$$
\sum \rho_{n}|\cos n s h|<\infty
$$

if $s$ is an odd number.

If we replace $s$ in (4) by an odd $q$, then we get

$$
\sum \rho_{n}=\sum \rho_{n}\left|\cos n q \cdot \frac{p}{q} \pi\right|<\infty
$$

which is the required inequality.

We may also prove the theorem geometrically by using the cited Fatou's theorem concerning the symmetry of points of absolute convergence, and the fact that if the series

$$
\sum \rho_{n} \cos \left(n x-\alpha_{n}\right)
$$

converges absolutely at a point $x^{\prime}$, then also it converges at the point $x^{\prime}+\pi$.

THEOREM 2. For a given sequence $\left\{\rho_{\nu}\right\}$ of positive numbers such that $\sum \rho_{p}=\infty$, and an irreducible fraction $p^{\prime} / q^{\prime}(\neq 0)$ with an even $q^{\prime}$ or an irrational number $\lambda$, we can construct a trigonometrical series

$$
\sum \rho_{\nu} \cos n_{r} x
$$

which is absolutely convergent at $x=p^{\prime} \cdot \pi / q^{\prime}$ or $x=\lambda \pi$ respectively.

Evidently the conjugate series $\sum \rho_{\nu} \sin n_{v} x$ converges absolutely at $x=0$.

For the proof of the first part of the theorem, we put

$$
n_{\nu} \equiv(2 \nu+1) q^{\prime} / 2 \quad(\nu=1,2, \cdots) ;
$$

then, $p^{\prime}$ being an odd number, we have

$$
\sum \rho_{\nu}\left|\cos n_{\nu} \frac{p^{\prime}}{q^{\prime}} \pi\right|=\sum \rho_{\nu}\left|\cos (2 \nu+1) p^{\prime} \frac{\pi}{2}\right|=0 .
$$

For the second part we consider the sequence

$$
\{n \lambda x\}_{n=1,2}, \ldots
$$

which is uniformly distributed in the interval $(0,2 \pi)$ by Weyl's theorem; then we can find a strictly increasing sequence $\left\{n_{\nu}\right\}$ such that 


$$
\left|\cos n_{\nu} \lambda \pi\right| \leqq 1 /\left(\sum_{\mu=1}^{\nu} \rho_{\mu}\right)^{2}
$$

Thus we have

$$
\sum_{\nu} \rho_{\nu}\left|\cos n_{\nu} \lambda \pi\right| \leqq \sum_{\nu} \frac{\rho_{\nu}}{\left(\sum_{\mu=1}^{\nu} \rho_{\mu}\right)^{2}}<\infty .^{2}
$$

THEOREM 3. Let $\sum \rho_{n}=\infty$, and one of the following conditions be satisfied:

(i) $\rho_{n}$ is non-increasing,

(ii) $\rho_{n} / \rho_{m}$ is bounded uniformly for $n \geqq m>0$,

(iii) $\sum_{v=1}^{n} 1 / \rho_{\nu}=O\left(n^{2}\right)$.

If $\sum \rho_{n} \cos \left(n x-\alpha_{n}\right)$ converges absolutely at $x=x_{0}$, then it does not converge elsewhere; moreover the conjugate series $\sum \rho_{n} \sin \left(n x-\alpha_{n}\right)$ does not converge anywhere.

The first part of the theorem is due to R. Salem. ${ }^{3}$ For the proof of the second part we suppose, if possible, that

$$
\sum \rho_{n} \sin \left(n x-\alpha_{n}\right)
$$

converges absolutely at $x=x_{1}$, then by (4)

$$
\sum \rho_{n}\left|\cos n\left(x_{0}-x_{1}\right)\right|<\infty
$$

from which we deduce that

$$
\sum \rho_{n}<\infty
$$

by the argument used by Salem to prove the first part. This is a contradiction.

2. Consider the trigonometrical series

$$
\sum \rho_{n} \cos \left(n x-\alpha_{n}\right) \quad\left(\rho_{n} \geqq 0 ; n=1,2, \cdots\right) .
$$

If $\sum \rho_{n}=\infty$, then the set of absolute convergence points of (5) is of measure zero by the Denjoy-Lusin theorem. On the other hand by Theorem 3, (iii), if $\sum_{\nu=1}^{n} 1 / \rho_{\nu}=O\left(n^{2}\right)$, the series (5) converges ab-

2 This proof is shown by Professor Izumi. We can easily find a sequence $\left\{n_{\nu}\right\}$ and a set $E$ of generalized Cantor type such that $\lambda_{\pi} \in E$ and $\left|\cos n_{\nu}\right|<1 /\left(\sum_{\mu-1}^{\nu} \rho_{\mu}\right)^{2}$ $(\nu=1,2, \cdots)$ for every $x \in E$, that is $\sum \rho_{\nu}\left|\cos n_{\nu} x\right|<\infty$, for every $x$ in $E$ which includes $\lambda \pi$. Therefore we see that each of the series $\sum \rho_{\nu} \cos n_{\nu} x$ and $\sum \rho_{\nu} \sin n_{\nu} x$ has the perfect set of absolute convergence points.

${ }^{3} \mathrm{R}$. Salem, The absolute convergence of trigonometrical series, Duke Math. J. vol. 8 (1941). 
solutely at most at one point. As a counterpart of this theorem we prove the following theorem.

TheOREM 4. If $\sum \rho_{n}=\infty$, and $\rho_{n}=O(1 / n)$, then the set $E$ of absolute convergence points of (5) is of $\alpha$-capacity $(0<\alpha<1)$ zero. ${ }^{4}$

For the proof, let $E$ be of positive $\alpha$-capacity $(0<\alpha<1)$; then by the Salem-Zygmund theorem, ${ }^{5}$ there exists positive distribution $\mu \in \operatorname{Lip} \alpha$ concentrated on $E$. If we put

$$
d \mu \sim \frac{1}{2 \pi}+\sum\left(a_{n} \cos n x+b_{n} \sin n x\right),
$$

then

$$
\mu(x)-\frac{x}{2 \pi}+C \sim \sum_{1}^{\infty} \frac{a_{n} \sin n x-b_{n} \cos n x}{n}
$$

and, $\mu(x)$ being monotone, the left-hand side is of bounded variation. Hence by a theorem due to Zygmund ${ }^{6}$ we have

$$
\sum\left(\left|a_{n}\right|+\left|b_{n}\right|\right) / n<\infty .
$$

Let us put $\mu(H) \equiv \int_{H} d \mu$ for $H \subseteq(0,2 \pi)$. By Egoroff's theorem, for any $\epsilon>0(\epsilon<1 / 6)$, there exist a set $\varepsilon \subseteq E$ and a positive number $M$ such that $\mu(\varepsilon)>1-\epsilon$ and

$$
\sum_{1}^{\infty} \rho_{n}\left|\cos \left(n x-\alpha_{n}\right)\right|<M
$$

Integrating both sides we get

$$
\sum_{n=1}^{\infty} \rho_{n} \int \varepsilon\left|\cos \left(n x-\alpha_{n}\right)\right| d \mu<M \mu(\varepsilon)
$$

where

4 R. Salem and A. Zygmund, Capacity of sets and Fourier series, Trans. Amer. Math. Soc. vol. 59 (1946) pp. 23-41. The $\alpha$-capacity of sets is defined as follows: The distribution of positive mass on the unit circle $|z|=1, z=r e^{i x}$, shall be supposed to be a non-negative additive function of sets, from which we define a point function $\mu(x)$ non-decreasing for $0 \leqq x \leqq 2 \pi$. It will be supposed that the total distribution $\mu(2 \pi)$ $-\mu(0)=1$. If for a (Borel) set $E, \int_{E} d_{\mu}=\int_{0}^{2 \pi} d \mu$, we say that a distribution $\mu$ is concentrated on $E$. A set $E$ is said to be of positive $\alpha$-capacity, if there exists a distribution $\mu$ concentrated on $E$ and $V \equiv \int_{0}^{2 \pi} d \mu(t) /\left|e^{i t}-r e^{i x}\right| \alpha$ is uniformly bounded in $x$ as $r \rightarrow 1$.

- Salem-Zygmund, loc. cit. footnote 4.

- Zygmund, loc. cit. footnote 1, p. 136. 


$$
\begin{aligned}
& 2 \int_{\varepsilon}\left|\cos \left(n x-\alpha_{n}\right)\right| d \mu \\
& \geqq \int \varepsilon^{\left(1-2 \cos 2 \alpha_{n} \cos 2 n x+\sin 2 \alpha_{n} \sin 2 n x\right) d \mu} \\
& \geqq 1-\epsilon+\cos 2 \alpha_{n} \int \varepsilon^{\cos 2 n x d \mu}+\sin 2 \alpha_{n} \int \varepsilon \sin 2 n x d \mu \\
& =1-\epsilon+\cos 2 \alpha_{n} \int_{E} \cos 2 n x d \mu+\sin 2 \alpha_{n} \int_{E} \sin 2 n x d \mu
\end{aligned}
$$

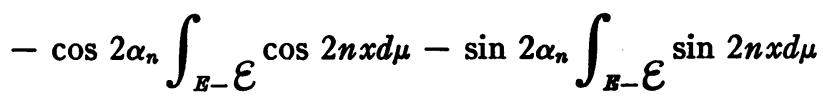

$$
\begin{aligned}
& \geqq 1-\epsilon+\cos 2 \alpha_{n} \int_{0}^{2 \pi} \cos 2 n x d \mu+\sin 2 \alpha_{n} \int_{0}^{2 \pi} \sin 2 n x d \mu-2 \epsilon \\
& \geqq 1-3 \epsilon-\left|a_{2 n}\right|-\left|b_{2 n}\right| \text {. }
\end{aligned}
$$

Consequently

$$
2 M \geqq \sum \rho_{n}(1-3 \epsilon)-\sum \rho_{n}\left(\left|a_{2 n}\right|+\left|b_{2 n}\right|\right) .
$$

On the other hand, by (2) and $\rho_{n}=O(1 / n), \sum \rho_{n}\left(\left|a_{2 n}\right|+\left|b_{2 n}\right|\right)$ $=O\left(\sum\left(\left|a_{2 n}\right|+\left|b_{2 n}\right|\right) / n\right)<\infty$ which contradicts (7).

TheOREM 5. If (5) is convergent absolutely on a set of positive $\alpha$-capacity $(0<\alpha<1)$, we can divide the suffixes into two classes $\left\{n_{i}\right\}$ and $\left\{m_{i}\right\}$ such that $\sum 1 / m_{i}<\infty, \sum \rho_{n_{i}}<\infty .{ }^{7}$

For, we get (6) by the hypothesis, and then we can deduce the theorem by the usilal argument.

REMARK. Let $\mu$ be a distribution concentrated on $E$. If

$$
\int_{0}^{2 \pi} \log \frac{1}{\left|e^{i t}-r e^{i x}\right|} d \mu(t)
$$

is uniformly bounded in $x$ as $r \rightarrow 1$, we say that $E$ is of positive logarithmic capacity. Salem ${ }^{8}$ gave an example such that $\sum \rho_{n}=\infty$ and $\sum \rho_{n} \cos \left(n x-\alpha_{n}\right)$ converges absolutely on a set of positive logarithmic capacity. But for $\alpha$-capacity such an example seems to be open.

\section{TôHOKU UNIVERSITY}

\footnotetext{
${ }^{7}$ For example, see Zygmund, Sur la caractère de convergence des series orthogonales,
} Matematica vol. 9 (1935) pp. 86-88.

8 Salem, loc. cit. footnote 3. 